\title{
ORAL METFORMIN VERSUS INSULIN IN TREATMENT OF GESTATIONAL DIABETES MELLITUS
}

Ali E. Ali, MD, Mohamed E. Mohamed, MD, Mostafa A. Ahmed, MD

Obstetrics and Gynecology Department, Faculty of Medicine, Zagazig University

\section{ABSTRACT}

Objective: The aim of this study is to compare the efficacy of metformin with that of insulin in treatment of gestational diabetes mellitus (GDM).

Subjects \& Methods: The study included 94 pregnant women who have been diagnosed as gestational diabetics at 25-33 weeks gestation with singleton pregnancy. They had fasting blood glucose (FBG) level ranging from $95-120 \mathrm{mg} / \mathrm{dl}$ or 2-hour postprandial blood glucose (PPBG) level ranging from 120-190 mg/dl. The exclusion criteria include pregnant women with preexisting DM and underlying diseases known to affect fetal growth or drug clearance. All patients were randomized to receive metformin $(n=47)$ or insulin $(n=47)$.

Results: There were no significant differences between the two groups regarding maternal age, gravidity, parity, GA at time of diagnosis, GA at beginning of treatment, and BMI at time of diagnosis. Additionally, it was noticed that women in the metformin treated group reached sooner to the glucose targets and maternal weight gain was less in the metformin treated group. It was found that women who required supplemental insulin had higher BMI, earlier gestational age at the start of treatment and higher levels of FBG and 2 hours glucose level at time of diagnosis.

Conclusion: Analysis of the results revealed that metformin was an effective medication for control of blood glucose in women with GDM who failed to achieve euglycemic with diet only.

Corresponding Auther:

Name : tarek ahmed ahmed ali

Email: Dr.santawy@gmail.com

Tel : 01004455429

\section{INTRODUCTION}

Gi estational diabetes mellitus is observed in seven to eighteen percentiles of pregnancies $^{(1)}$. It is defined as any level of glucose intolerance with onset or first recognition during pregnancy ${ }^{(2)}$.

Common complications include a higher rate of neonatal hypoglycemia, cesarean section, preeclampsia and fetal macrosomia, all of which are substantially decreased when glucose levels are controlled, either by diet and exercise primarily or by treatment when the first approach failed ${ }^{(3)}$.

Rule treatment to obtain adequate glucose levels is insulin therapy. However, insulin needs various insulin injections daily, which affect patient commitment. Moreover, its higher price may prevent treatment for a lot of patients ${ }^{(4)}$. Oral antidiabetic agents have been investigated as an alternative therapy during pregnancy because of their lower cost and ease of use ${ }^{(5)}$.

Metformin is a hypoglycemic agent that increases peripheral insulin sensitivity and reduces hepatic gluconeogenesis. Recently, a lot of studies have investigated the use of metformin for the treatment of gestational diabetes mellitus and two randomized clinical trials have shown the same neonatal results, concluding that metformin seems to be an effective alternative for the treatment of gestational diabetes mellitus. However, response to treatment in patients with GDM is highly dependent on characteristics of the patient ${ }^{(6)}$.

\section{PATIENTS AND METHODS}

This two armed prospective randomized controlled clinical trial was conducted prospectively on 94 pregnant women who selected from the patients attending the outpatient clinic of the Obstetrics and Gynecology Department, Faculty of Medicine, Zagazig University Hospitals. The study included 94 pregnant women with GDM classified into two groups Forty seven patient in each group using open EPI at power $80 \%$ and confidence interval $95 \%$.

\section{Inclusion criteria:}

1. Patients diagnosed as gestational diabetes $>24$ weeks gestation

\section{Singleton pregnancy}

3. Patients failed to achieve adequate glycemic control on diet and exercise for a minimum period of 1 week.

\section{Exclusion criteria:}


1. Risk factors for lactic acidosis (renal failure, heart failure, chronic liver disease, severe chronic pulmonary disease, coronary insufficiency and history of thromboembolic phenomena).

2. Underlying diseases known to affect fetal growth or drug clearance such as severe chronic hypertension, thyroid disease, chronic renal insufficiency, hepatic disease, thrombophilia, systemic lupus erythromatosis and history of intrauterine growth retardation.

3. Anatomic and chromosome anomalies of the conceptus detected by ultrasonography.

4. Hypersensitivity to metformin.

5. Patients concurrently on anticoagulants.

6. Women who have contraindication to take metformin

7. Non-compliant patients.

\section{All patients were subjected to the following:}

History: Personal history including: Name, age, sex, residence and occupation.

Past history: previous medical illness or drug intake, previous pregnancy and mode of delivery.

Clinical Examination: Careful general examination including body weight, height, blood pressure and lower limb edema. Maternal body mass index (BMI) was calculated using the earliest available body weight (the weight in kilograms divided by the square of the height in meters. Abdominal examination for assessment of estimated fetal weight, fetal movement.

\section{Laboratory investigations:}

- Initial investigation:

- Glycosylated hemoglobin (HbA1c).

- Fasting and postprandial blood glucose level.

- Liver and renal function tests.

- Routine pregnancy care investigation (CBC, urine analysis).

- Follow up investigation:

- Every patient asked to attend to the outpatient clinic every two weeks until 28 weeks gestational age, then every week until delivery.

- At each visit, the patient was asked to do FBG and 2-hour PPBG level.

- HbA1C will be assessed every 3 months.
Ultrasonography:

Trans-Abdominal ultrasound assessment for congenital anomalies, liquor volume and growth 24-40 weeks.

\section{Management plan:}

A diagnosis of GDM at the time of study was established when the patient presented 2 or more altered results on the oral glucose tolerance test with $100 \mathrm{~g}$ or $75 \mathrm{~g}$ glucose. After the diagnosis of GDM the women were referred for follow-up to an outpatient clinic. For nutritional counseling, a calorie intake of 25 to $35 \mathrm{kcal} / \mathrm{kg}$ weight per day was recommended depending on the classification of the pregestational body mass index (BMI) of the patient. In addition, the calories were divided to comprise $55 \%$ carbohydrates, $15 \%$ proteins, and $30 \%$ lipids. A 30-minutes' walk, 3 times a week, was recommended. Unsatisfactory glycemic control was defined among patients who presented more than $30 \%$ of capillary glycemia results above the reference values 1 week after commencing diet therapy combined with physical activity ${ }^{(7)}$.

Medication based treatment was then initiated. At this time, patients who met all inclusion criteria were divided randomly to receive either metformin (study group) or insulin (control group) according to an electronic randomization list. Data were collected weekly during return visits.

\section{Insulin group}

Subjects were received human NPH insulin (intermediate-acting insulin). The starting dose 0.9 units per kg body weight per day, with half the dose being administered in the morning (before breakfast), 1/4 of the total dose before lunch, and $1 / 4$ at 22:00 hours. This group was asked to monitor glucose 7 times per day (at fasting, 2 hours after breakfast, 1hour before lunch, 2 hours after lunch, 1 hour before dinner, 2 hours after dinner and at 3 in the morning). The doses were adjusted weekly to achieve adequate glycemic control. If preprandial glucose levels were normal and postprandial glucose levels were high, regular insulin will added half an hour before that meal in addition to NPH insulin ${ }^{(8)}$.

\section{Metformin group}


Metformin was started at dose of 500 $\mathrm{mg}$ and increased up to $2550 \mathrm{mg}$ in 3 divided doses as tolerated until glycemic control was achieved. Target blood glucose levels for glycemic control were FBS $100 \mathrm{mg} / \mathrm{dl}$ and PPBG $126 \mathrm{mg} / \mathrm{dl}$. If blood glucose levels were higher than the cut off values 1-2 weeks after treatment or at any time during treatment with maximum dose of metformin, insulin was added as supplementary treatment with metformin ${ }^{(9)}$.

\section{Neonatal assessment:}

The newborn baby will be assessed by a senior pediatrician for:

- APGAR score (1 - 5 min).

- Umbilical cord PH

- Blood glucose level

- Neonatal weight
- $\mathrm{RDS}$

- Neonatal hyperbilirubinemia

\section{Ethical issues:}

1) An official permission was obtained from the manager of outpatient clinic for helping in conduction of this work.

2) Obtaining verbal or written consent from each respondent before the interview.

\section{RESULTS}

Ten patients showed bad response to the highest dose of metformin $(2500 \mathrm{mg})$ received additional doses of insulin until control was achieved.

Nine patients didn't continue the follow up in our study and so excluded, the final analysis included 85 patients.

Table (1): Demographic data of both insulin and metformin groups.

\begin{tabular}{lcccc}
\hline & Insulin & Metformin & T/Z test & P-Value \\
\hline Age (years) & $31.34 \pm 3.62$ & $30.4 \pm 3.78$ & 1.224 & 0.224 \\
\hline Gravidity & $4(1-7)$ & $4(1-7)$ & 0.624 & 0.534 \\
\hline Parity & $2(0-5)$ & $2(0-6)$ & 0.068 & 0.946 \\
\hline G.A at diagnosis (weeks) & $28.02 \pm 1.49$ & $27.87 \pm 1.67$ & 0.454 & 0.651 \\
\hline G.A at established control & $30.02 \pm 1.49$ & $29.87 \pm 1.67$ & 0.454 & 0.651 \\
\hline BMI Kg/m ${ }^{2}$ (at diagnosis) & $30.85 \pm 2.42$ & $30.1 \pm 2.7$ & 1.406 & 0.163 \\
\hline HA1c\% (At diagnosis) & $6.29 \pm 0.58$ & $6.41 \pm 0.42$ & -1.185 & 0.239 \\
\hline FBS & $115.08 \pm 9.7$ & $113.61 \pm 9.6$ & 0.735 & 0.464 \\
\hline 2 hr PPBS & $178.63 \pm 6.83$ & $178.1 \pm 9.47$ & 0.312 & 0.756 \\
\hline
\end{tabular}

Table (2): Comparison between insulin group and metformin group as regard fasting and $2 \mathrm{~h}$ PPBG after establishment of control

\begin{tabular}{lcccc}
\hline & Insulin & Metformin & $\mathrm{t}$ & $\mathrm{P}$. value \\
\hline After treatment (ttt) FBS & $94.32 \pm 6.5$ & $95.16 \pm 9.06$ & 0.821 & 0.452 \\
\hline After ttt 2hr PPBS & $116.79 \pm 6.56$ & $120.76 \pm 9.13$ & 1.903 & 0.068 \\
\hline Before delivery FBS & $80.51 \pm 6.14$ & $82.42 \pm 5.21$ & 1.106 & 0.112 \\
\hline Before delivery 2hr PPBS & $106.16 \pm 6.76$ & $109.0 \pm 7.8$ & 1.054 & 0.103 \\
\hline
\end{tabular}

Table (3): Comparison between insulin group and metformin group as regard neonatal outcomes

\begin{tabular}{lcccc}
\hline & Insulin & Metformin & $\mathrm{t}$ & $\mathrm{P}$. value \\
\hline Birth Weight & $3957.7 \pm 188.6$ & $4098 \pm 221.2$ & -1.221 & 0.121 \\
\hline APGAR1 & $7.79 \pm 0.96$ & $7.97 \pm 0.71$ & -1.005 & 0.318 \\
\hline APGAR5 & $9.27 \pm 0.76$ & $9.52 \pm 0.67$ & -1.565 & 0.121 \\
\hline
\end{tabular}


Table (4): Comparison between insulin group and metformin group as regard complication distribution

\begin{tabular}{|c|c|c|c|c|c|c|c|}
\hline & & & \multicolumn{2}{|c|}{ Group } & \multirow{2}{*}{ Total } & \multirow{2}{*}{$\chi^{2}$} & \multirow{2}{*}{$\mathrm{P}$} \\
\hline & & & Insulin & Metformin & & & \\
\hline \multirow[t]{4}{*}{ Preeclampsia } & \multirow{2}{*}{ No } & $\mathrm{N}$ & 29 & 25 & 54 & \multirow{4}{*}{0.57} & \multirow{4}{*}{0.44} \\
\hline & & $\%$ & 67.4 & 59.5 & 63.5 & & \\
\hline & \multirow{2}{*}{ Yes } & $\mathrm{N}$ & 14 & 17 & 31 & & \\
\hline & & $\%$ & 32.6 & 40.5 & 36.5 & & \\
\hline \multirow[t]{4}{*}{ Polyhydramnios } & \multirow{2}{*}{ No } & $\mathrm{N}$ & 37 & 38 & 75 & \multirow{4}{*}{0.402} & \multirow{4}{*}{0.52} \\
\hline & & $\%$ & 86.0 & 90.5 & 88.2 & & \\
\hline & \multirow{2}{*}{ Yes } & $\mathrm{N}$ & 6 & 4 & 10 & & \\
\hline & & $\%$ & 14.0 & 9.5 & 11.8 & & \\
\hline \multirow[t]{4}{*}{ IUFD } & \multirow{2}{*}{ No } & $\mathrm{N}$ & 40 & 40 & 80 & \multirow{4}{*}{0.18} & \multirow{4}{*}{0.66} \\
\hline & & $\%$ & 93.0 & 95.2 & 94.1 & & \\
\hline & \multirow{2}{*}{ Yes } & $\mathrm{N}$ & 3 & 2 & 5 & & \\
\hline & & $\%$ & 7.0 & 4.8 & 5.9 & & \\
\hline \multirow[t]{4}{*}{ Macrosomia } & \multirow{2}{*}{ No } & $\mathrm{N}$ & 40 & 38 & 78 & \multirow{4}{*}{1.32} & \multirow{4}{*}{0.25} \\
\hline & & $\%$ & 95.2 & 88.4 & 91.8 & & \\
\hline & \multirow{2}{*}{ Yes } & $\mathrm{N}$ & 2 & 5 & 7 & & \\
\hline & & $\%$ & 4.8 & 11.6 & 8.2 & & \\
\hline \multirow[t]{4}{*}{ Congenital anomaly } & \multirow{2}{*}{ No } & $\mathrm{N}$ & 41 & 41 & 82 & \multirow{4}{*}{0.32} & \multirow{4}{*}{0.57} \\
\hline & & $\%$ & 95.3 & 97.6 & 96.5 & & \\
\hline & \multirow{2}{*}{ Yes } & $\mathrm{N}$ & 2 & 1 & 3 & & \\
\hline & & $\%$ & 4.7 & 2.4 & 3.5 & & \\
\hline \multirow[t]{2}{*}{ Total } & & $\mathrm{N}$ & 43 & 42 & 85 & & \\
\hline & & $\%$ & 100 & 100 & 100 & & \\
\hline
\end{tabular}

Table (5): Comparison between insulin group and metformin group as regard mode of delivery

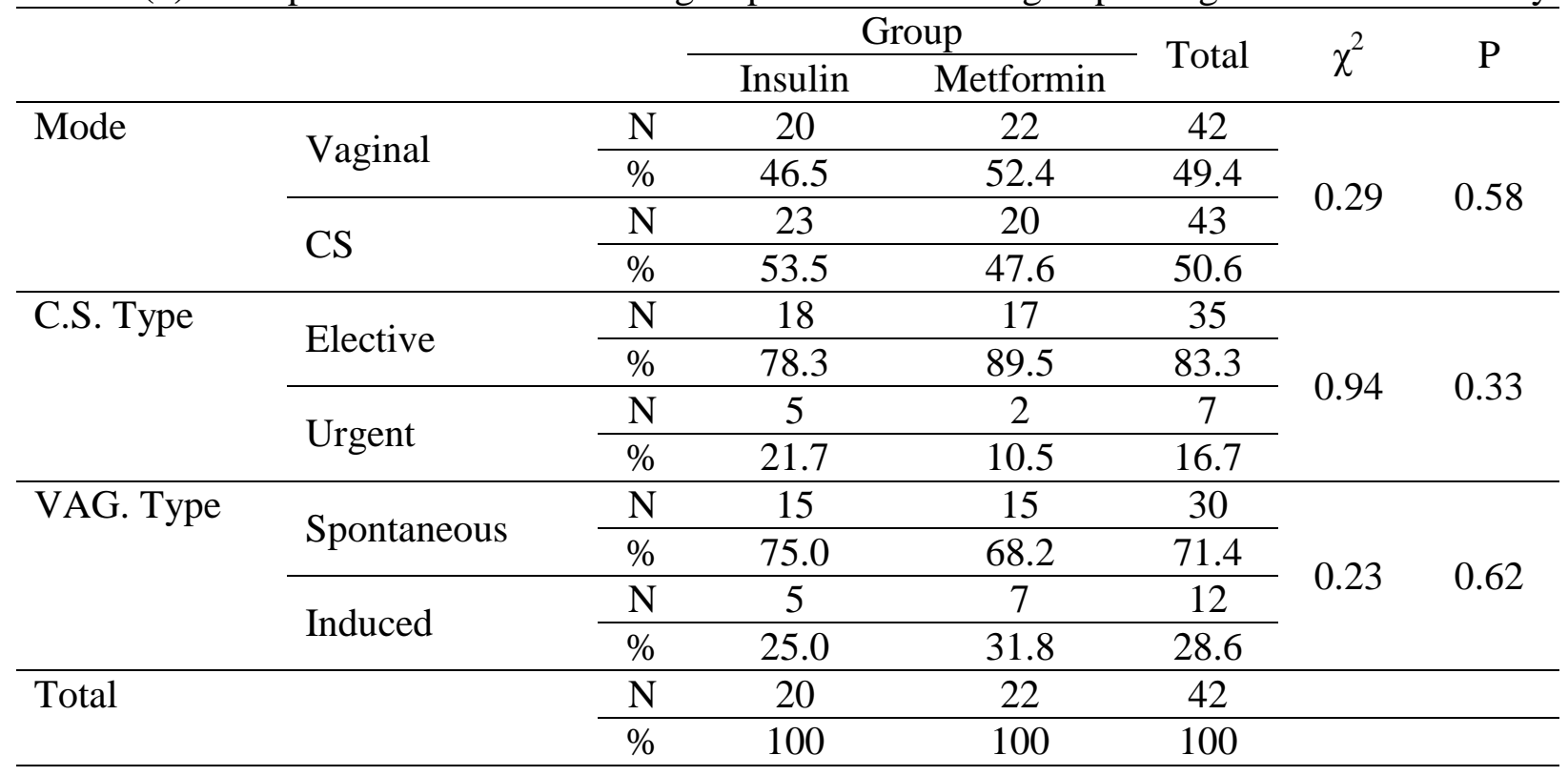


Table (6): Comparison of metformin group according to dose

\begin{tabular}{ccc}
\hline Metformin dose & $\mathrm{N}$ & $\%$ \\
\hline 1000 & 4 & 12.5 \\
\hline 1500 & 10 & 23.8 \\
\hline 2500 & 18 & 56.2 \\
\hline With insulin & 10 & 23.8 \\
\hline
\end{tabular}

Table (7): Comparisons between patient who received metformin only and patient who received metformin plus insulin as regard patient demographic data

\begin{tabular}{lcccc}
\hline & Metformin only & $\begin{array}{c}\text { Metformin } \\
\text { insulin }\end{array}$ & $\mathrm{t}$ & $\mathrm{P}$ \\
\hline Age (years) & $31.1 \pm 3.58$ & $28.5 \pm 4.01$ & 1.628 & 0.082 \\
\hline G.A at diagnosis & $27.78 \pm 1.84$ & $28.2 \pm 1.03$ & -0.681 & 0.499 \\
\hline G.A at established control & $29.78 \pm 1.84$ & $30.2 \pm 1.03$ & -0.681 & 0.499 \\
\hline BMI & $28.62 \pm 1.43$ & $34.2 \pm 0.42$ & -12.062 & $0.00^{* *}$ \\
\hline HA1c\% & $6.2 \pm 0.31$ & $7.0 \pm 0.09$ & -7.756 & $0.00^{* *}$ \\
\hline F.B.S. & $109.31 \pm 7.9$ & $125.2 \pm 4.68$ & -6.006 & $0.00^{* *}$ \\
\hline 2hr P.P.B.S. & $174.4 \pm 7.27$ & $189.8 \pm 6.57$ & -5.963 & $0.00^{* *}$ \\
\hline
\end{tabular}

** Very highly significant $(\mathrm{P}<0.001)$

Table (8): Comparison between metformin only group and metformin plus insulin group as regard mode of delivery

\begin{tabular}{|c|c|c|c|c|c|c|c|}
\hline & & & \multicolumn{2}{|c|}{ Group } & \multirow[b]{2}{*}{ Total } & \multirow[b]{2}{*}{$\chi^{2}$} & \multirow[b]{2}{*}{$\mathrm{P}$} \\
\hline & & & $\begin{array}{l}\text { Metformin } \\
\text { only }\end{array}$ & $\begin{array}{c}\text { Metformin } \\
+ \text { Insulin }\end{array}$ & & & \\
\hline \multirow[t]{4}{*}{$\mathrm{CS}$} & \multirow{2}{*}{ Vaginal } & $\mathrm{N}$ & 16 & 6 & 22 & \multirow{4}{*}{0.3} & \multirow{4}{*}{0.58} \\
\hline & & $\%$ & 50.0 & 60.0 & 52.4 & & \\
\hline & \multirow{2}{*}{$\mathrm{CS}$} & $\mathrm{N}$ & 16 & 4 & 20 & & \\
\hline & & $\%$ & 50.0 & 40.0 & 47.6 & & \\
\hline \multirow[t]{4}{*}{ CSTYPE } & \multirow{2}{*}{ Elective } & $\mathrm{N}$ & 13 & 4 & 17 & \multirow{4}{*}{0.59} & \multirow{4}{*}{0.44} \\
\hline & & $\%$ & 86.7 & 100 & 89.5 & & \\
\hline & \multirow{2}{*}{ Urgent } & $\mathrm{N}$ & 2 & 0 & 2 & & \\
\hline & & $\%$ & 13.3 & 0.0 & 10.5 & & \\
\hline \multirow[t]{4}{*}{ VAGTYPE } & \multirow{2}{*}{ Spontaneous } & $\mathrm{N}$ & 10 & 5 & 15 & \multirow{4}{*}{0.87} & \multirow{4}{*}{0.35} \\
\hline & & $\%$ & 62.5 & 83.3 & 68.2 & & \\
\hline & \multirow{2}{*}{ Induced } & $\mathrm{N}$ & 6 & 1 & 7 & & \\
\hline & & $\%$ & 37.5 & 16.7 & 31.8 & & \\
\hline
\end{tabular}

$\mathrm{P}>0.05=$ non-significant 
Table (9): Comparison between metformin only group and metformin plus insulin group as regard fasting and $2 \mathrm{hr}$ PPBG after establishment of control

\begin{tabular}{lcccc}
\hline & Metformin only & $\begin{array}{c}\text { Metformin } \\
\text { insulin }\end{array}$ & $\mathrm{t}$ & $\mathrm{p}$ \\
\hline After ttt fasting & $88.78 \pm 9.16$ & $90.4 \pm 9.1$ & -0.488 & 0.628 \\
\hline After ttt 2hr PPBG & $114.75 \pm 8.01$ & $118 \pm 12$ & -1.45 & 0.092 \\
\hline Before delivery fasting & $75.18 \pm 5.08$ & $76.2 \pm 5.82$ & -0.531 & 0.598 \\
\hline Before delivery 2hr PPBG & $103.87 \pm 7.55$ & $108.6 \pm 7.87$ & -1.710 & 0.095 \\
\hline $\mathrm{P}>0.05$ = non-significant & & & &
\end{tabular}

Table (10): Comparison between metformin only and metformin plus insulin group as regard neonatal outcome

\begin{tabular}{lcccc}
\hline & Metformin alone & $\begin{array}{c}\text { Metformin }+ \\
\text { insulin }\end{array}$ & $\mathrm{t}$ & $\mathrm{P}$ \\
\hline Birth Weight & $3939.06 \pm 204.6$ & $4105.0 \pm 305.0$ & -1.982 & 0.054 \\
\hline APGAR 1 Min. & $8.09 \pm 0.64$ & $7.6 \pm 0.84$ & 1.971 & 0.056 \\
\hline APGAR 5 Min. & $9.68 \pm 0.47$ & $9.32 \pm 0.94$ & 1.758 & 0.073 \\
\hline
\end{tabular}

Table (11): Comparison between metformin only group and metformin plus insulin group as regard complication

\begin{tabular}{|c|c|c|c|c|c|c|c|}
\hline & & & & & & & \\
\hline & & & $\begin{array}{l}\text { Metformin } \\
\text { alone }\end{array}$ & $\begin{array}{l}\text { With } \\
\text { insulin }\end{array}$ & Total & $X^{2}$ & $P$ value \\
\hline Preeclampsia & No & $\mathrm{N}$ & 19 & 6 & 25 & & \\
\hline & NO & $\%$ & 59.4 & 60.0 & 59.5 & م $\cap \Omega 1$ & 007 \\
\hline & & $\mathrm{N}$ & 13 & 4 & 17 & 0.001 & 0.91 \\
\hline & Yes & $\%$ & 40.6 & 40.0 & 40.5 & & \\
\hline Polyhydramnios & No & $\mathrm{N}$ & 28 & 10 & 38 & & \\
\hline & No & $\%$ & 87.5 & 100 & 90.5 & 138 & 024 \\
\hline & Yes & $\mathrm{N}$ & 4 & 0 & 4 & 1.38 & 0.24 \\
\hline & Yes & $\%$ & 12.5 & 0.0 & 9.5 & & \\
\hline IUFD & No & $\mathrm{N}$ & 30 & 10 & 40 & & \\
\hline & No & $\%$ & 93.8 & 100 & 95.2 & 065 & 041 \\
\hline & & $\mathrm{N}$ & 2 & 0 & 2 & 0.65 & 0.41 \\
\hline & Yes & $\%$ & 6.2 & 0.0 & 4.8 & & \\
\hline Macrosomia & No & $\mathrm{N}$ & 32 & 5 & 37 & & \\
\hline & No & $\%$ & 100 & 50.0 & 88.1 & 101 & $\cap 0 \cap * *$ \\
\hline & Yes & $\mathrm{N}$ & 0 & 5 & 5 & 18.1 & $0.00^{\mathrm{N}+\mathrm{T}}$ \\
\hline & Yes & $\%$ & 0.0 & 50.0 & 11.9 & & \\
\hline Congenital anomaly & & $\mathrm{N}$ & 32 & 9 & 41 & & \\
\hline & No & $\%$ & 100 & 90.0 & 97.6 & 327 & 007 \\
\hline & Yec & $\mathrm{N}$ & 0 & 1 & 1 & 3.21 & 0.01 \\
\hline & Yes & $\%$ & 0.0 & 10.0 & 2.4 & & \\
\hline Total & & $\mathrm{N}$ & 32 & 10 & 42 & & \\
\hline & & $\%$ & 100 & 100 & 100 & & \\
\hline
\end{tabular}

$\mathrm{P}>0.05=$ non-significant

$\mathrm{P}<0.001=$ Very highly significant 
Table (12): Patients characters distribution among different metformin doses group

\begin{tabular}{|c|c|c|c|c|c|c|c|c|}
\hline & & $\mathrm{N}$ & Mean & $\pm \mathrm{SD}$ & Min & Max & $\mathrm{F}$ & P. value \\
\hline \multirow[t]{4}{*}{ Age (years) } & 1000 & 4 & 29.0000 & 4.00000 & 31.00 & 39.00 & \multirow{4}{*}{1.029} & \multirow{4}{*}{0.112} \\
\hline & 1500 & 10 & 31.8000 & 2.39444 & 26.00 & 32.00 & & \\
\hline & 2500 & 18 & 30.0556 & 2.91996 & 27.00 & 35.00 & & \\
\hline & +insulin & 10 & 30.9000 & 4.01248 & 25.00 & 38.00 & & \\
\hline \multirow{4}{*}{$\begin{array}{l}\text { G.A. at } \\
\text { diagnosis }\end{array}$} & 1000 & 4 & 26.0000 & 2.00000 & 25.00 & 29.00 & \multirow{4}{*}{2.037} & \multirow{4}{*}{0.125} \\
\hline & 1500 & 10 & 27.9000 & 1.85293 & 26.00 & 30.00 & & \\
\hline & 2500 & 18 & 28.1111 & 1.67644 & 25.00 & 31.00 & & \\
\hline & + insulin & 10 & 28.2000 & 1.03280 & 27.00 & 30.00 & & \\
\hline \multirow[t]{4}{*}{ G.A ttt } & 1000 & 4 & 28.0000 & 2.00000 & 27.00 & 31.00 & \multirow{4}{*}{2.037} & \multirow{4}{*}{0.125} \\
\hline & 1500 & 10 & 29.9000 & 1.85293 & 28.00 & 32.00 & & \\
\hline & 2500 & 18 & 30.1111 & 1.67644 & 27.00 & 33.00 & & \\
\hline & + insulin & 10 & 30.2000 & 1.03280 & 29.00 & 32.00 & & \\
\hline \multirow[t]{4}{*}{ BMI } & 1000 & 4 & 28.0000 & 0.00000 & 28.00 & 28.00 & \multirow{4}{*}{50.391} & \multirow{4}{*}{$0.00 * *$} \\
\hline & 1500 & 10 & 28.3000 & 1.05935 & 27.00 & 30.00 & & \\
\hline & 2500 & 18 & 28.9444 & 1.69679 & 27.00 & 32.00 & & \\
\hline & + insulin & 10 & 34.2000 & 0.42164 & 34.00 & 35.00 & & \\
\hline \multirow[t]{4}{*}{ HA1c\% } & 1000 & 4 & 6.0750 & 0.15000 & 6.00 & 6.30 & \multirow{4}{*}{26.135} & \multirow{4}{*}{$0.00 * *$} \\
\hline & 1500 & 10 & 6.0500 & 0.19579 & 5.90 & 6.30 & & \\
\hline & 2500 & 18 & 6.3278 & 0.35114 & 5.90 & 6.80 & & \\
\hline & + insulin & 10 & 7.0000 & 0.09428 & 6.90 & 7.10 & & \\
\hline \multirow[t]{4}{*}{ F.B.S. } & 1000 & 4 & 105.7500 & 1.50000 & 105.0 & 108.00 & \multirow{4}{*}{15.514} & \multirow{4}{*}{$0.00 * *$} \\
\hline & 1500 & 10 & 105.8000 & 7.13053 & 97.00 & 115.00 & & \\
\hline & 2500 & 18 & 112.0556 & 8.26383 & 98.00 & 122.00 & & \\
\hline & + insulin & 10 & 125.2000 & 4.68568 & 118.0 & 129.00 & & \\
\hline \multirow[t]{4}{*}{ HBS2 } & 1000 & 4 & 162.0000 & 2.00000 & 161.0 & 165.00 & \multirow{4}{*}{53.556} & \multirow{4}{*}{$0.00 * *$} \\
\hline & 1500 & 10 & 170.0000 & 2.82843 & 167.0 & 177.00 & & \\
\hline & 2500 & 18 & 179.6111 & 3.91286 & 173.0 & 184.00 & & \\
\hline & + insulin & 10 & 189.8000 & 6.57943 & 178.0 & 195.00 & & \\
\hline
\end{tabular}

$\mathrm{P}>0.05=$ non-significant

$\mathrm{P}<0.001=$ Very highly significant

Table (13): Effect of treatment comparison and distribution among different metformin doses group

\begin{tabular}{|c|c|c|c|c|c|c|c|c|}
\hline & & $\mathrm{N}$ & Mean & $\pm \mathrm{SD}$ & Min & Max & $\mathrm{F}$ & $\mathrm{P}$ \\
\hline \multirow{4}{*}{$\begin{array}{l}\text { After ttt } \\
\text { FBS }\end{array}$} & 1000 & 4 & 75.0000 & 2.00000 & 74.00 & 78.00 & \multirow{4}{*}{8.845} & \multirow{4}{*}{$0.00 * *$} \\
\hline & 1500 & 10 & 85.1000 & 8.64677 & 73.00 & 95.00 & & \\
\hline & 2500 & 18 & 93.8889 & 5.69715 & 84.00 & 102.00 & & \\
\hline & + insulin & 10 & 90.4000 & 9.10677 & 79.00 & 101.00 & & \\
\hline \multirow{4}{*}{$\begin{array}{l}\text { After ttt } \\
2 \mathrm{hr} \text { PPBG }\end{array}$} & 1000 & 4 & 109.0000 & 8.00000 & 105.00 & 121.00 & \multirow{4}{*}{2.931} & \multirow{4}{*}{$0.046^{*}$} \\
\hline & 1500 & 10 & 110.8000 & 5.07280 & 105.00 & 120.00 & & \\
\hline & 2500 & 18 & 118.2222 & 7.90797 & 109.00 & 132.00 & & \\
\hline & + insulin & 10 & 119.0000 & 11.98147 & 99.00 & 131.00 & & \\
\hline \multirow{4}{*}{$\begin{array}{l}\text { Before } \\
\text { delivery } \\
\text { FBS }\end{array}$} & 1000 & 4 & 72.7500 & 1.50000 & 72.00 & 75.00 & \multirow{4}{*}{0.615} & \multirow{4}{*}{0.610} \\
\hline & 1500 & 10 & 74.5000 & 3.62859 & 70.00 & 81.00 & & \\
\hline & 2500 & 18 & 76.1111 & 6.10582 & 70.00 & 90.00 & & \\
\hline & + insulin & 10 & 76.2000 & 5.82714 & 69.00 & 82.00 & & \\
\hline \multirow{2}{*}{$\begin{array}{l}\text { Before } \\
\text { delivery }\end{array}$} & 1000 & 4 & 98.0000 & 6.00000 & 95.00 & 107.00 & \multirow{2}{*}{3.717} & \multirow{2}{*}{$0.019 *$} \\
\hline & 1500 & 10 & 100.8000 & 4.10420 & 91.00 & 105.00 & & \\
\hline
\end{tabular}




\begin{tabular}{|c|c|c|c|c|c|c|c|c|}
\hline \multirow[t]{2}{*}{$2 \mathrm{hr}$ PPBS } & 2500 & 18 & 106.8889 & 8.08695 & 98.00 & 121.00 & & \\
\hline & + insulin & 10 & 108.6000 & 7.87683 & 95.00 & 118.00 & & \\
\hline \multirow{4}{*}{$\begin{array}{l}\text { G.A. at } \\
\text { delivery }\end{array}$} & 1000 & 4 & 39.0000 & .00000 & 39.00 & 39.00 & \multirow{4}{*}{1.862} & \multirow{4}{*}{0.153} \\
\hline & 1500 & 10 & 38.3000 & .82327 & 37.00 & 39.00 & & \\
\hline & 2500 & 18 & 38.2778 & .75190 & 37.00 & 39.00 & & \\
\hline & + insulin & 10 & 37.8000 & 1.22927 & 36.00 & 39.00 & & \\
\hline \multirow{4}{*}{$\begin{array}{l}\text { Weight } \\
\text { gain }\end{array}$} & 1000 & 4 & 3.2500 & .50000 & 3.00 & 4.00 & \multirow{4}{*}{1.334} & \multirow{4}{*}{0.278} \\
\hline & 1500 & 10 & 3.5000 & .52705 & 3.00 & 4.00 & & \\
\hline & 2500 & 18 & 3.2778 & .89479 & 2.00 & 5.00 & & \\
\hline & + insulin & 10 & 3.8000 & .42164 & 3.00 & 4.00 & & \\
\hline
\end{tabular}

$\mathrm{P}>0.05=$ non-significant, ${ }^{*} \mathrm{P}<0.05=$ significant, $* * \mathrm{P}<0.01=$ highly significant

Table (14): Neonatal outcome distribution among different metformin doses group.

\begin{tabular}{|c|c|c|c|c|c|c|c|c|}
\hline & & $\mathrm{N}$ & Mean & $\pm \mathrm{SD}$ & Min & Max & $\mathrm{F}$ & $\mathrm{P}$ \\
\hline \multirow{4}{*}{$\begin{array}{l}\text { Birth } \\
\text { Weight }\end{array}$} & 1000 & 4 & 3925.00 & 350.00000 & 3750 & 4450 & \multirow{4}{*}{1.520} & \multirow{4}{*}{0.225} \\
\hline & 1500 & 10 & 3890.00 & 157.76213 & 3700 & 4200 & & \\
\hline & 2500 & 18 & 3969.44 & 197.88506 & 3650 & 4250 & & \\
\hline & + insulin & 10 & 4105.00 & 305.00455 & 3850 & 4600 & & \\
\hline \multirow{4}{*}{$\begin{array}{l}\text { APGAR } \\
1\end{array}$} & 1000 & 4 & 8.0000 & .00000 & 8.00 & 8.00 & \multirow{4}{*}{2.420} & \multirow{4}{*}{0.081} \\
\hline & 1500 & 10 & 7.8000 & .42164 & 7.00 & 8.00 & & \\
\hline & 2500 & 18 & 8.2778 & .75190 & 7.00 & 9.00 & & \\
\hline & + insulin & 10 & 7.6000 & .84327 & 7.00 & 9.00 & & \\
\hline \multirow{4}{*}{$\begin{array}{l}\text { APGAR } \\
5\end{array}$} & 1000 & 4 & 9.7500 & .50000 & 9.00 & 10.00 & \multirow{4}{*}{11.585} & \multirow{4}{*}{$\begin{array}{c}0.00^{*} \\
*\end{array}$} \\
\hline & 1500 & 10 & 9.1000 & .31623 & 9.00 & 10.00 & & \\
\hline & 2500 & 18 & 10.000 & .00000 & 10.00 & 10.00 & & \\
\hline & + insulin & 10 & 9.0000 & .94281 & 8.00 & 10.00 & & \\
\hline
\end{tabular}

$\mathrm{P}>0.05=$ non-significant

$* * \mathrm{P}<0.001=$ Very highly significant

Table (15): Complication distribution among different metformin doses group

\begin{tabular}{|c|c|c|c|c|c|c|c|c|c|}
\hline & & & \multicolumn{4}{|c|}{ Groups } & \multirow{2}{*}{ Total } & \multirow{2}{*}{$X^{2}$} & \multirow{2}{*}{$\mathrm{P}$} \\
\hline & & & 1000 & 1500 & 2500 & + insulin & & & \\
\hline \multirow[t]{4}{*}{ Preeclampsia } & \multirow{2}{*}{ No } & $\mathrm{N}$ & 2 & 4 & 13 & 6 & 25 & \multirow{4}{*}{2.93} & \multirow{4}{*}{0.41} \\
\hline & & $\%$ & 50.0 & 40.0 & 72.2 & 60.0 & 59.5 & & \\
\hline & \multirow{2}{*}{ Yes } & $\mathrm{N}$ & 2 & 6 & 5 & 4 & 17 & & \\
\hline & & $\%$ & 50.0 & 60.0 & 27.8 & 40.0 & 40.5 & & \\
\hline \multirow[t]{4}{*}{ Polyhydramnios } & \multirow{2}{*}{ No } & $\mathrm{N}$ & 3 & 9 & 16 & 10 & 38 & \multirow{4}{*}{2.22} & \multirow{4}{*}{0.52} \\
\hline & & $\%$ & 75.0 & 90.0 & 88.9 & 100 & 90.5 & & \\
\hline & \multirow{2}{*}{ Yes } & $\mathrm{N}$ & 1 & 1 & 2 & 0 & 4 & & \\
\hline & & $\%$ & 25.0 & 10.0 & 11.1 & 0.0 & 9.5 & & \\
\hline \multirow[t]{4}{*}{ IUFD } & \multirow{2}{*}{ No } & $\mathrm{N}$ & 3 & 9 & 18 & 10 & 40 & \multirow{4}{*}{5.61} & \multirow{4}{*}{0.13} \\
\hline & & $\%$ & 75.0 & 90.0 & 100 & 100 & 95.2 & & \\
\hline & \multirow{2}{*}{ Yes } & $\mathrm{N}$ & 1 & 1 & 0 & 0 & 2 & & \\
\hline & & $\%$ & 25.0 & 10.0 & 0.0 & 0.0 & 4.8 & & \\
\hline
\end{tabular}




\begin{tabular}{|c|c|c|c|c|c|c|c|c|c|}
\hline \multirow[t]{4}{*}{ Macrosomia } & \multirow{2}{*}{ No } & $\mathrm{N}$ & 4 & 10 & 18 & 5 & 37 & \multirow{4}{*}{6.72} & \multirow{4}{*}{0.081} \\
\hline & & $\%$ & 100 & 100 & 100 & 50.0 & 88.1 & & \\
\hline & \multirow{2}{*}{ Yes } & $\mathrm{N}$ & 0 & 0 & 0 & 5 & 5 & & \\
\hline & & $\%$ & 0.0 & 0.0 & 0.0 & 50.0 & 11.9 & & \\
\hline \multirow[t]{4}{*}{ Congenital anomaly } & \multirow{2}{*}{ No } & $\mathrm{N}$ & 4 & 10 & 18 & 9 & 41 & \multirow{4}{*}{3.27} & \multirow{4}{*}{0.35} \\
\hline & & $\%$ & 100 & 100 & 100 & 90.0 & 97.6 & & \\
\hline & \multirow{2}{*}{ Yes } & $\mathrm{N}$ & 0 & 0 & 0 & 1 & 1 & & \\
\hline & & $\%$ & 0.0 & 0.0 & 0.0 & 10.0 & 2.4 & & \\
\hline \multirow{2}{*}{ Total } & & $\mathrm{N}$ & 4 & 10 & 18 & 10 & 42 & & \\
\hline & & $\%$ & 100 & 100 & 100 & 100 & 100 & & \\
\hline
\end{tabular}

Table (16): Insulin dose comparison between metformin group and insulin only group

Insulin $\underset{\text { Insulin }}{\text { Metformin }}+\quad \mathrm{t} \quad$ P-Value

$\begin{array}{llll}\text { Dose of insulin in unit } \quad 39.5 \pm 10.2 & 32.8 \pm 8.7 & 2.021 & 0.042 *\end{array}$

\section{DISCUSSION}

The management of GDM is important because appropriate therapy can decrease many of its adverse pregnancy outcomes. Effective treatment regimens consist of dietary therapy, exercise, self-blood glucose monitoring, and administration of insulin if target blood glucose values are not met with diet regulation alone ${ }^{(10)}$.

Standard medical treatment to achieve adequate glucose levels is insulin therapy. However, this therapy requires multiple daily injections, which may reduce patient compliance; furthermore its high cost may preclude treatment for some patients. A safe and effective oral agent would offer advantages over insulin and may well prove more acceptable to patients ${ }^{(11)}$.

Metformin is a biguanide hypoglycemic agent that reduces hepatic gluconeogenesis and increases peripheral insulin sensitivity is a rational option for women with GDM. Evidence from the Metformin in Gestational Diabetes (MiG) trial showed that, compared with insulin, metformin was not associated with increased prenatal complications although there was an increase in spontaneous preterm births. When asked to choose, metformin was preferred to insulin by GDM women ${ }^{(12) .}$

A recent metanalysis of six large studies, outside Egypt, has shown that the use of oral hypoglycemic agents (OHAs) in treating GDM was not associated with neonatal hypoglycemia, macrosomia or increased incidence of cesarean section ${ }^{(6)}$.

The present study was conducted to evaluate the effectiveness and safety of metformin in treating patients with GDM in Egypt. The Egyptian woman is different in culture as regards commitment to medicine and examinations courses, partially also due to the high personal cost of treatment. This may make it easier to give her oral drug (and reduce the need to daily glucose monitoring) rather than injectable drugs. Also, the cost of metformin is cheaper than the cost of insulin.

Metformin is a biguanide hypoglycemic agent that reduces hepatic gluconeogenesis and increases peripheral insulin sensitivity is a rational option for women with GDM. Evidence from the Metformin in Gestational Diabetes (MiG) trial showed that, compared with insulin, metformin was not associated with increased prenatal complications although there was an increase in spontaneous preterm births. When asked to choose, metformin was preferred to insulin by GDM women ${ }^{(12)}$.

Concerning patients' characteristics in both groups, there were no significant differences between the two groups regarding maternal age (in metformin treated group $30.4 \pm 3.78$ versus $31.34 \pm 3.62$ in the insulin 
treated group, $\mathrm{p}=0.224$ ), gravidity, parity, GA at time of diagnosis (in metformin treated group $27.87 \pm 1.67$ weeks versus $28.02 \pm 1.49$ weeks in insulin treated group, $p=0.651$ ), GA at the establishment of control (in metformin treated group $29.87 \pm 1.67$ weeks versus $30.02 \pm 1.49$ weeks in insulin treated group, $\mathrm{p}=0$. 651), BMI at the time of diagnosis (in metformin treated group $30.1 \pm 2.7 \mathrm{~kg} / \mathrm{m}^{2}$ versus $30.85 \pm 2.42 \mathrm{~kg} / \mathrm{m}^{2}, \quad \mathrm{p}=0.163$, and $\mathrm{HbA1c}$ at time of diagnosis (in metformin treated group $6.41 \pm 0.42 \%$ versus $6.29 \pm 0.58$ in insulin treated group $\%, p=0.239$ ).

This was in agreement with the study of Rowan et al. ${ }^{(12)}$ who reported that there were no significant differences between the two groups as regards patients' characteristics this agreement might be due to the similarity in inclusion criteria and study design between our study and the study of Rowan et al. On the other hand, the study of Spaulonci et al. (8) reported that there was a significant difference in the number of pregnancies between groups with a median number of 2 pregnancies in metformin treated group versus 3 pregnancies in insulin treated group. This difference might result from the various ethnic groups and entry criteria as maternal age was slightly older.

With respect to glycemic control, no significant difference in mean pre-treatment glucose levels was observed between the two groups (fasting glucose levels were $113.61 \pm 9.6 \mathrm{mg} / \mathrm{dl}$ in metformin treated group versus $115.08 \pm 9.7 \mathrm{mg} / \mathrm{dl}$ in insulin treated group, $\mathrm{p}=0.464$ and 2-hours postprandial glucose levels were $178.1 \pm 9.47$ in metformin treated group versus $178.63 \pm 6.83 \mathrm{mg} / \mathrm{dl}$ in insulin treated group, $\mathrm{p}=0.756$ ).

However, after introduction of the drugs, the average postprandial glycemic levels during the first week after randomization were just significantly lower in the metformin treated group.

These values did not differ significantly between 2 groups in the last 2 weeks before delivery, a finding suggesting that glucose targets were reached sooner in the metformin group.

This was in agreement with the studies of Rowan et al. ${ }^{(12)}$ and Shirin et al. ${ }^{(13)}$ who reported that the postprandial glycemic levels at the first week after randomization were significantly lower in the metformin treated group $(117.0 \pm 16.2 \mathrm{mg} / \mathrm{dl}$ versus $120.6 \pm 18$ $\mathrm{mg} / \mathrm{dl}$ in insulin treated group). The likely explanation is that it takes time for the patients to master the usage and dosecalculation of insulin. Our results were not in agreement with Moore et al. (5) which revealed that the fasting and 2-hour postprandial glucose levels were not statistically different between insulin and metformin group. In both groups the fasting values were $<100 \mathrm{mg} / \mathrm{dl} \quad(\mathrm{p}=0.400)$ and $2-$ hour postprandial glucose levels all averaged $<120 \mathrm{mg} / \mathrm{dl}$ in both groups $(\mathrm{p}=0.545)$. They considered any postprandial glucose level below $120 \mathrm{mg} / \mathrm{dl}$ to be normal irrespective of its exact value.

Concerning the gestational age at time of delivery, the insulin versus metformin groups did not show significant difference. GA, at time of delivery, in the metformin treated group was $27.87 \pm 1.67$ weeks and in the insulin treated group was $28.02 \pm 1.49$ weeks, $p=0.651$. Also there was no difference in the rate of cesarean section between the two groups. In the metformin treated group, the ratio of C.S were (47.6\%), while in insulin treated group were $(53.5 \%), \mathrm{p}=0.58$. This was in agreement with studies of Tertti et al. (14), Michael et al. ${ }^{(15),}$ but not in agreement with the study of Rowan et al. ${ }^{(12)}$ who reported that the average gestational ages at delivery were significantly lower in the metformin group ( $\mathrm{p}=0.001)$ and preterm birth rate was significantly more common in the metformin group. This inconsistency may be due to chance or unrecognized effect of metformin on the labor. On the contrary, Balani et al. ${ }^{(16)}$ showed that preterm delivery was more common in the insulin treated group, but the study of balani et al was merely a casecontrol study.

Our study showed that BMI, HA1c, FBS and 2hr PPBS were significantly higher in metformin patients who received additional doses of insulin.

In the study performed by Rowan et al. (12), the BMI, HbA1C at time of diagnosis, elevated fasting and post-prandial glycemia before introduction of medication all predicted the need for supplemental insulin. 
On the other hand, the study of Spaulonci et al. ${ }^{(8)}$ reported that the BMI and $\mathrm{HbA1C}$ at time of diagnosis were similar in the 2 groups. Neither BMI nor glycated hemoglobin influenced insulin need. These disparities are probably explained by differences in population studied because diabetes and glycemic control vary widely between different populations.

In the present study, there was a borderline significance in the difference between both groups as regards fetal birth weight. Average birth weights were slightly lower in the metformin treated group. There were 5 fetuses $(11.6 \%)$ with macrosomia in the metformin treated group, and 2 fetuses $(4.8 \%)$ in insulin group. The pooled results showed no significant difference between the two groups as regards the rate of large for gestational age (LGA) and small for gestational age (SGA) newborns. This was in agreement with the study of Moore et al. ${ }^{(5)}$ $\mathrm{p}=0.039$, and Tertti et al. ${ }^{(14)} \mathrm{p}=0.038$, but not in agreement with the study of Spaulonci et al. (8) who reported that there were no significant difference between 2 groups regarding newborn weight (in metformin group $3143.7 \pm 446.6$ gmvs $3237.6 \pm 568.8$ gm in insulin group, $\mathrm{p}=0.390$ ).

Concerning 1-min Apgar score there was no significant difference between the 2 groups with $\mathrm{p}=0.318,5$-min Apgar score also there was no significant difference between the 2 groups with $\mathrm{p}=0.121$ ).

As regards the birth defect rates, there was no significant difference between 2 groups. In the insulin group, one neonate had a ventricular septal defect (VSD); and in the metformin treated group, one neonate had a unilateral cleft lip. No cases of perinatal deaths occurred in this study. This result was expected since the evidence supporting the safe use of metformin in pregnancy is available from studies in patients with polycystic ovary syndrome (PCOS) ${ }^{(17) .}$

In order to characterize and identify mothers needing insulin in addition to metformin, we compared the subgroups of metformin only. As regards patients' characteristics, women requiring supplemental insulin had a higher BMI at the time of diagnosis with $\mathrm{p}<0.001$. The group requiring supplemental insulin also had a higher mean glucose level during the last week before introduction of medication.

The blood glucose level, one week after starting drug treatment, was higher postprandially in the metformin group requiring supplemental insulin.

This was in agreement with studies of Rowan et al. ${ }^{(12)}$ and Spaulonci et al ${ }^{(8)}$ who reported that the fasting and post-prandial glucose levels were higher in the metformin requiring supplemental insulin group.

Our study showed that BMI,HA1c\%, FBS and $2 \mathrm{hr}$ P.P.B.S were significantly higher in patients who received insulin and metformin when compared to patients who received different doses of metformin $(1000-$ 1500 - 2500). As Regard comparison between different metformin dose groups we found that APGAR 5 score significantly higher in 1000 group and 2500 group than other groups.

As regards economic costs the insulin treatment was more costly than metformin treatment in terms of drug price, cost of blood tests and follow-up, cost of syringes used for insulin.

On the national level, the use of metformin as an alternative to insulin in treating GDM may save the country a lot of money spent either in support or importing insulin.

The findings of our study suggest that metformin is an effective and safe treatment option for women with GDM. Metformin is comparable with insulin in glycemic control, providing additional evidence for the use of metformin in GDM. The results of this study show no significant difference in the risk of maternal or perinatal adverse outcomes with the use of metformin compared to insulin in treating GDM. This study shows the potential advantages of metformin over insulin in cost, similar glucose level after control, faster reaching ideal glucose levels, patient compliance, easier use, maternal weight gain and neonatal birth weight adjusted for gestational age.

In addition, a subgroup of women more likely to require supplemental insulin for adequate glycemic control was identified; a 
finding that might be useful when choosing a drug for the treatment of GDM.

From our results it had concluded that approximately $80 \%$ of women with GDM could be successfully and safely treated with metformin when diet therapy and exercise fail to reduce blood glucose values sufficiently. Moreover, it is observed that metformin is not associated with increased risk of adverse pregnancy outcomes.

The current study indicates that women clearly preferred metformin therapy over insulin where it offers a simpler, faster acting, cheaper and more convenient alternative to insulin in such individuals, but we need to do a lot of studies on a large scale of pregnant women suffering of GDM to support this data.

\section{REFERENCES}

1. Coustan DR, Lowe LP, Metzger BE, and Dyer AR: The Hyperglycemic and Adverse Pregnancy Outcome (HAPO) study: paving the way for new diagnostic criteria for gestational mellitus. Am. J. Obstet. Gynecol. 2010; 202: 654-661.

2. American Diabetes Association: Diagnosis and classification of diabetes mellitus. Diabetes Care; 201033: 562.

3. Langer O, Yogen Y, Most O: Gestational diabetes the consequences of no treating. Am.

J. Obstet. Gynecol.; 2005192: 989-997.

4. Standards of medical care in diabetes: Diabetes Care; 35(Suppl 1): 2012s11-63.

5. Moore LE, Briery CM, Clokey D, Martin RW, Williford NJ, et al.: Metformin and insulin in the management of gestational diabetes mellitus: preliminary results of a comparison. $\mathbf{J}$ Reprod Med; 2007:52: 1011-1015.

6. Dhulkotia JS, Ola B, Fraser R, Farrell T: Oral hypoglycemic agents vs insulin in management of gestational diabetes: a systematic review and meta-analysis. Am J Obstet Gynecol; 2010:203: 457 e451-459.

7. Metzgar BE, Buchanan TA, Coustan DR, Deleiva A, Dunger DB, Hadden DR, Hod M, Kitzmiller JL, Kjos SL, Oats JN, Pettitt DA, Sacks DA, Zoupas C: Summary and Recommendations of the Fifth International
Workshop-Conference on Gestational Diabetes. Diabetes Care; 30 (Suppl 2): 2007:25-33.

8. Spaulonci CP, Bernardes LS, Trindade TC, et al.: Randomized trial of metformin vs insulin in the management of gestational diabetes. Am J Obstet Gynecol; 2013:209: 34.e1-7.

9. Jahanara Ainuddin, Nasim Karim, Anjum Ara Hasan, Sanower Ali: Metformin versus insulin treatment in gestational diabetes in pregnancy in a developing country. A randomized control trial. Diabetes research and clinical practice; 107: 2015:290-299.

10. Khattab S, Mohsen IA, Foutouh IA, Ramadan A, Moaz Kim C.: Gestational diabetes: risks, management, and treatment options. Int $\mathbf{J}$ Women's Health 2: 2010:339-351.

11. Hayer L and Shehata HA: Gestational diabetes mellitus. Curr Obstet Gynecol; 15: 2005:368374.

12. Rowan JA, Haque WM, Gao W: Metformin vs insulin for the treatment of gestational diabetes. The New England Journal of Medicine; 2008:358: 2003-2015.

13. Shirin N, Azin A, Fatemah RS, Nooshin A, Sanaz M, Sohela AK: Metformin compared with insulin in the management of gestational diabetes mellitus: A randomized clinical trial. Diabetes research and Clinical practice: website2012www.elseiver.com/locate/diabres.

14. Tertti K, Ekblad U, Koskinen P, Vahlberg T, RonnemaaT: Metformin vs. insulin in gestational diabetes. A randomized study characterizing metformin patients needing additional insulin. Diabetes Obstet Metab; 2012:15: 246-251.

15. Micheal J, Paglia M, Coustan D: The use of oral antidiabetics medication in gestational diabetes mellitus. Current Diabetes Reports; 2009:9: 287-290.

16. Balani J, Hayer SL, Rodin DA, Shehata H: Pregnancy outcomes in women with gestational diabetes treated with metformin or insulin: a case-control study. Diabet Med; 2009:26: 798-802.

17. McCarthy E, Walker S, McLachlan K: Metformin in obstetric and gynecologic practice: a review. Obst Gyn Surv; 2004:59: 118-127. 